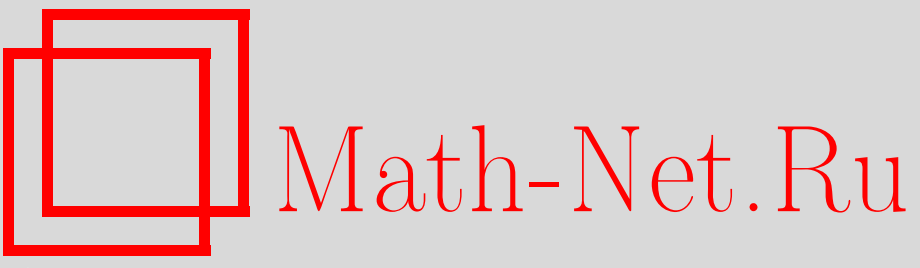

V. E. Mosyagin, Exact asymptotics for the distribution of the time of attaining the maximum for a trajectory of a compound Poisson process with linear drift, Mat. Tr., 2019, Volume 22, Number 2, 134-156

DOI: https://doi.org/10.33048/mattrudy.2019.22.208

Use of the all-Russian mathematical portal Math-Net.Ru implies that you have read and agreed to these terms of use

http://www.mathnet.ru/eng/agreement

Download details:

IP: 18.234 .156 .22

April 26, 2023, 11:27:15 


\title{
ТОЧНАЯ АСИМПТОТИКА РАСПРЕДЕЛЕНИЯ МОМЕНТА ДОСТИЖЕНИЯ МАКСИМУМА ТРАЕКТОРИЕЙ ОБОБЩЕННОГО ПРОЦЕССА ПУАССОНА С ЛИНЕЙНЫМ СНОСОМ
}

\author{
B. Е. Мосягин
}

\begin{abstract}
При определенных условиях на параметры $a, p, q$ изучается функция распределения $G(x)$ момента достижения максимума траекторией случайного процесса $a t-\nu_{+}(p t)+\nu_{-}(-q t), t \in(-\infty, \infty)$, где $\nu_{-}(t)$ и $\nu_{+}(t)$ - независимые стандартные пуассоновские процессы при $t \geq 0$ и равные нулю при $t<0$. В работе найдена точная асимптотика хвостов распределения $G(x)$. Отмечается связь рассматриваемой задачи с задачей оценивания неизвестной точки разрыва плотности распределения по известной выборке.
\end{abstract}

Ключевые слова и фразы: пуассоновский процесс с линейным сносом, оценивание точки разрыва плотности распределения, точная асимптотика распределений.

\section{§1. Введение и постановка задачи}

Пусть $\nu_{-}(t)$ и $\nu_{+}(t)$ обозначают независимые стандартные процессы Пуассона при $t \geq 0$ и доопределенные нулем при $t<0$.

Определим случайный процесс

$$
Y(t)=a t-\nu_{+}(p t)+\nu_{-}(-q t), \quad t \in(-\infty, \infty),
$$

параметры $a, p, q>0$ которого удовлетворяют соотношениям

$$
p>q, \quad a=\frac{p-q}{\ln (p / q)} .
$$

Из равенства (2) и теоремы Лагранжа выводим неравенства

$$
p>a=\frac{p-q}{\ln p-\ln q}>q,
$$

обеспечивающие процессу (1) отрицательный средний снос

$$
\mathbb{E} Y(t)=\left\{\begin{array}{l}
(a-p) t<0, \quad t>0, \\
(a-q) t<0, \quad t<0 .
\end{array}\right.
$$

(C) В. Е. Мосягин; 2019 
Отсюда и из $[14, \S 26]$ следует существование единственной (с вероятностью 1) собственной случайной величины $t^{*}(Y)=\operatorname{argmax}\{Y(t)\}$ с непрерывной функцией распределения $G(x)$, аналитический вид которой был найден в [10] и с некоторыми уточнениями в [11].

Определим положительную при $z \neq 1$ функцию

$$
\Lambda(z)=z-1-\ln z, \quad z>0,
$$

заметив, что в силу равенства (2)

$$
\Lambda(p / a)=\Lambda(q / a)
$$

и это значение будем в дальнейшем обозначать через $\lambda$.

В работе [5] для хвостов распределения $G(x)$

$$
\mathbb{P}\left(t^{*}(Y)<-x\right)=G(-x) \quad \text { и } \quad \mathbb{P}\left(t^{*}(Y)>x\right)=1-G(x)
$$

получены точные асимптотические формулы

$$
\begin{aligned}
G(-x) & \sim \frac{c_{-}(p, q)}{x^{3 / 2}} e^{-\lambda a x}, \\
1-G(x) & \sim \frac{c_{+}(p, q)}{x^{3 / 2}} e^{-\lambda a x},
\end{aligned}
$$

где константы $c_{ \pm}$зависят только от параметров процесса (1). При этом в [5] отмечается, что найти в явном виде одну из этих констант не удается, а другая может быть получена, если воспользоваться аналитическим аппаратом, приведенным в [3; 4].

Цель настоящей статьи состоит в нахождении явного вида констант $c_{ \pm}$, а также в получении ряда результатов, уточняющих аналитический вид функции распределения $G(x)$.

В заключение параграфа отметим, что процессы вида (1) возникают в страховой математике [13, с. 719], а также в математической статистике $[1 ; 2 ; 6-9]$ в задачах об оценке неизвестной точки разрыва плотности распределения по известной выборке. Действительно, если плотность $f(x, \theta)$, $\theta \in \Theta$, абсолютно непрерывного распределения имеет единственный скачок в точке $x=\theta$ :

$$
0<q(\theta)=f(\theta-0, \theta)<f(\theta+0, \theta)=p(\theta), \quad \theta \in \Theta,
$$

a $\left\{\widehat{\theta}_{n}\right\}$ - это оценки максимального правдоподобия (ОМП) истинного параметра $\theta_{0}$, то при $n \rightarrow \infty$ предельное распределение нормированных ОМП $n\left(\widehat{\theta}_{n}-\theta_{0}\right)$ совпадает с распределением момента достижения максимума траекторией процесса (см. [6, гл. 5$])$ :

$$
Z(t)=\left(p\left(\theta_{0}\right)-q\left(\theta_{0}\right)\right) t-\ln \left(p\left(\theta_{0}\right) / q\left(\theta_{0}\right)\right)\left(\nu_{+}\left(p\left(\theta_{0}\right) t\right)-\nu_{-}\left(-q\left(\theta_{0}\right) t\right)\right) .
$$


Если здесь положим $p\left(\theta_{0}\right)=p, q\left(\theta_{0}\right)=q$, то $Z(t)=\ln (p / q) Y(t)$, а значит, $t^{*}(Z)=t^{*}(Y)$.

\section{§2. Основные предположения и результаты}

В целях упрощения формулировок утверждений и их доказательств сделаем в процессе (1) линейную замену времени $t:=a t$, приводящую к процессу

$$
Y^{*}(t)=t-\nu_{+}(p t)+\nu_{-}(-q t), \quad t \in(-\infty, \infty),
$$

в котором $p:=p / a, q:=q / a$. Тогда соответствующие процессу (7) условия (2)-(3) преобразуются к виду

$$
\begin{aligned}
p>a & =1>q>0, \\
p-q & =\ln (p / q),
\end{aligned}
$$

где последнее равенство представим в более удобной для дальнейшего применения форме

$$
p e^{-p}=q e^{-q} .
$$

Обозначим через $G^{*}(x)$ функцию распределения момента достижения максимума траекторией процесса (7). Тогда в силу (6) при $x \rightarrow \infty$ верны асимптотические формулы

$$
\begin{aligned}
G^{*}(-x) & \sim \frac{c_{-}^{*}(p, q)}{x^{3 / 2}} e^{-\lambda x}, \\
1-G^{*}(x) & \sim \frac{c_{+}^{*}(p, q)}{x^{3 / 2}} e^{-\lambda x},
\end{aligned}
$$

где (см. (4-5))

$$
\lambda=\Lambda(p)=\Lambda(q) .
$$

Константы $c_{ \pm}^{*}$ из (10) будут найдены из анализа аналитических выражений для распределений $G^{*}(-x)$ и $G^{*}(x)$ при $x>0$, полученных в [10, теорема 3$]$ и [11, теорема 1]:

$$
\begin{gathered}
G^{*}(-x)=(1-q) q\left(\int_{x}^{\infty} \sum_{k=[z]+1}^{\infty} \pi_{k}(q) d z-\int_{x}^{\infty} e^{b z} \sum_{k=[z]+1}^{\infty} \pi_{k}(q) e^{-b k} d z\right) \\
G^{*}(x)=\frac{(p-1) q}{(p-q)}+\beta \int_{0}^{x} e^{-p z} \sum_{k=0}^{[z]} \frac{p^{k} z^{k-1}}{k !}(z-k) \psi(z-k) d z
\end{gathered}
$$

где

$$
\begin{aligned}
\pi_{k}(q) & =(q k)^{k-1} e^{-q k} / k !, \quad k=1,2, \ldots \\
b & =\beta(1-q / p)
\end{aligned}
$$


$\beta$ - единственный положительный корень уравнения

$$
1-e^{-\beta}=\beta / p
$$

a $[z]$ - целая часть числа $z$. Функция $\psi(\cdot)$ под интегралом в (13) является функцией распределения:

$$
\begin{aligned}
\psi(x) & =\mathbb{P}\left(\sup _{t<0} Y^{*}(t) \leqslant x\right) \\
& =(1-q) \sum_{m=0}^{[x]}(-1)^{m} q^{m} \frac{(x-m)^{m}}{m !} e^{q(x-m)}, \quad x \geq 0,
\end{aligned}
$$

аналитический вид которой найден в [14, с. 167], а ее свойства изучены в $[10$, леммы 2,4$]$.

Следующие теоремы являются основными результатами работы.

Теорема 1. Константа $c_{-}^{*}$ в асимптотическом представлении (10) имеет вид

$$
c_{-}^{*}=\frac{(1-q) q e^{1-q}}{\sqrt{2 \pi}\left(1-q e^{1-q}\right)}\left(\frac{1}{1-q e^{1-q}}-\frac{p\left(\exp \left\{(p-q)^{2} / p\right\}-1\right)}{(p-q)^{2}\left(\exp \left\{(p-q)^{2} / p\right\}-q e^{1-q}\right)}\right) .
$$

Теорема 2. Константа $c_{+}^{*}$ в асимптотическом представлении (10) имеет вид

$$
c_{+}^{*}=\frac{p(1-q)}{\sqrt{2 \pi}(p-1)^{2}}\left(\frac{1}{1-q e^{1-q}}+\frac{\left(p^{2}-p q+q\right) \exp \{q(1-p) / p\}-1}{(p \exp \{q(1-p) / p\}-1)^{2}}\right) .
$$

\section{§3. Доказательства}

Лемма 1. Уравнение (15) имеет корень $\beta=p-q$, а константа $b$ из (12) и (14) равна $(p-q)^{2} / p$.

Доказательство. Подстановка $\beta=p-q>0$ в уравнение (15) приводит его к соотношению (9).

Лемма 2. Функция распределения (12) при любом натуральном значении $n$ представима в виде

$$
G^{*}(-n)=(1-q) q\left(\sum_{k=1}^{\infty} k \pi_{k+n}(q)-\frac{1}{b} \sum_{k=1}^{\infty}\left(1-e^{-b k}\right) \pi_{k+n}(q)\right)
$$

где последовательность $\left\{\pi_{k}(q)\right\}$ определена в (14), а константа b в лемме 1. 
Доказательство. Введем обозначения для интегралов из (12):

$$
A_{n}=\int_{n}^{\infty} \sum_{k=[z]+1}^{\infty} \pi_{k}(q) d z, \quad B_{n}=\int_{n}^{\infty} e^{b z} \sum_{k=[z]+1}^{\infty} \pi_{k}(q) e^{-b k} d z .
$$

Тогда

$$
\begin{gathered}
G^{*}(-n)=(1-q) q\left(A_{n}-B_{n}\right), \\
A_{n}=\sum_{m=n}^{\infty} \int_{m}^{m+1} \sum_{k=[z]+1}^{\infty} \pi_{k}(q) d z=\sum_{m=n}^{\infty} \sum_{k=m+1}^{\infty} \pi_{k}(q) \\
=\sum_{k=n+1}^{\infty} \sum_{m=n}^{k-1} \pi_{k}(q)=\sum_{k=n+1}^{\infty}(k-n) \pi_{k}(q)=\sum_{k=1}^{\infty} k \pi_{k+n}(q) .
\end{gathered}
$$

Подобным образом находим

$$
\begin{aligned}
B_{n} & =\sum_{m=n}^{\infty} \int_{m}^{m+1} e^{b z} \sum_{k=[z]+1}^{\infty} \pi_{k}(q) e^{-b k} d z=\sum_{m=n}^{\infty} \sum_{k=m+1}^{\infty} \pi_{k}(q) e^{-b k} \int_{m}^{m+1} e^{b z} d z \\
& =\sum_{k=n+1}^{\infty} \pi_{k}(q) e^{-b k} \sum_{m=n}^{k-1} \int_{m}^{m+1} e^{b z} d z=\sum_{k=n+1}^{\infty} \pi_{k}(q) e^{-b k} \int_{n}^{k} e^{b z} d z \\
& =\sum_{k=n+1}^{\infty} \pi_{k}(q) e^{-b k} \frac{e^{b k}-e^{b n}}{b}=\frac{1}{b} \sum_{k=1}^{\infty} \pi_{k+n}(q)\left(1-e^{-b k}\right) .
\end{aligned}
$$

Подстановка окончательных выражений для $A_{n}$ и $B_{n}$ в $(18)$ завершает доказательство.

Лемма 3. При $n \rightarrow \infty$ имеем

$$
\begin{aligned}
\sum_{k=1}^{\infty} \pi_{k+n}(q) & \sim \frac{c_{-}^{(1)}}{\sqrt{2 \pi} q n^{3 / 2}} e^{-\lambda n}, \\
\sum_{k=1}^{\infty} e^{-b k} \pi_{k+n}(q) & \sim \frac{c_{-}^{(2)}}{\sqrt{2 \pi} q n^{3 / 2}} e^{-\lambda n}, \\
\sum_{k=1}^{\infty} k \pi_{k+n}(q) & \sim \frac{c_{-}^{(3)}}{\sqrt{2 \pi} q n^{3 / 2}} e^{-\lambda n},
\end{aligned}
$$

где $c_{-}^{(1)}=e^{-\lambda} /\left(1-e^{-\lambda}\right), c_{-}^{(2)}=e^{-\lambda} /\left(e^{b}-e^{-\lambda}\right), c_{-}^{(3)}=e^{-\lambda} /\left(1-e^{-\lambda}\right)^{2}$, а константа $\lambda$ определена в (11). 
Доказательство. Преобразуем последовательность (14) с привлечением определений (4) и (11) следующим образом:

$$
\pi_{k}(q)=\frac{k^{k-1} e^{-k}}{q k !} e^{-(q-1-\ln q) k}=\frac{k^{k-1} e^{-k}}{q k !} e^{-\lambda k}, \quad k=1,2, \ldots
$$

Тогда

$$
\pi_{k+n}(q)=\frac{e^{-\lambda n}}{\sqrt{2 \pi} q n^{3 / 2}} C_{k}(n) e^{-\lambda k}
$$

где

$$
C_{k}(n)=\frac{\sqrt{2 \pi} n^{3 / 2}(k+n)^{k+n-1} e^{-(k+n)}}{(k+n) !}, \quad k=1,2, \ldots
$$

Следовательно,

$$
\sum_{k=1}^{\infty} \pi_{k+n}(q)=\frac{e^{-\lambda n}}{\sqrt{2 \pi} q n^{3 / 2}} \sum_{k=1}^{\infty} C_{k}(n) e^{-\lambda k} .
$$

Применяя формулу Стирлинга $(k+n) ! \sim \sqrt{2 \pi}(k+n)^{k+n+1 / 2} e^{-(k+n)}, n \rightarrow \infty$, к последовательности (20), находим

$$
\lim _{n \rightarrow \infty} C_{k}(n)=1, \quad k=1,2, \ldots,
$$

а так как $(k+n) !>\sqrt{2 \pi}(k+n)^{k+n+1 / 2} e^{-(k+n)}$, то для всех $k$ и $n$ получаем

$$
C_{k}(n)<\frac{n^{3 / 2}}{(k+n)^{3 / 2}}<1 .
$$

Последние обстоятельства приводят к следующей цепочке равенств:

$$
\lim _{n \rightarrow \infty} \sum_{k=1}^{\infty} C_{k}(n) e^{-\lambda k}=\sum_{k=1}^{\infty} \lim _{n \rightarrow \infty} C_{k}(n) e^{-\lambda k}=\sum_{k=1}^{\infty} e^{-\lambda k}=c_{-}^{(1)} .
$$

Отсюда и из (21) получаем первую асимптотическую формулу леммы. Вторая и третья формулы доказываются аналогично из ключевого выражения (19). Например,

$$
\begin{gathered}
\sum_{k=1}^{\infty} k \pi_{k+n}(q)=\frac{e^{-\lambda n}}{\sqrt{2 \pi} q n^{3 / 2}} \sum_{k=1}^{\infty} C_{k}(n) k e^{-\lambda k} \\
\lim _{n \rightarrow \infty} \sum_{k=1}^{\infty} C_{k}(n) k e^{-\lambda k}=\sum_{k=1}^{\infty} k e^{-\lambda k}=e^{-\lambda} /\left(1-e^{-\lambda}\right)^{2}=c_{-}^{(3)} .
\end{gathered}
$$


Доказательство теоремы 1. Из представления (17) и леммы 3 получаем асимптотическое выражение

$$
G^{*}(-n) \sim \frac{1-q}{\sqrt{2 \pi}}\left(c_{-}^{(3)}-\frac{c_{-}^{(1)}-c_{-}^{(2)}}{b}\right) \frac{e^{-\lambda n}}{n^{3 / 2}}, \quad n \rightarrow \infty .
$$

Отсюда находим константу

$$
\begin{aligned}
c_{-}^{*} & =\frac{1-q}{\sqrt{2 \pi}}\left(\frac{e^{-\lambda}}{\left(1-e^{-\lambda}\right)^{2}}-\frac{1}{b}\left(\frac{e^{-\lambda}}{1-e^{-\lambda}}-\frac{e^{-\lambda}}{e^{b}-e^{-\lambda}}\right)\right) \\
& =\frac{(1-q) e^{-\lambda}}{\sqrt{2 \pi}\left(1-e^{-\lambda}\right)}\left(\frac{1}{1-e^{-\lambda}}-\frac{e^{b}-1}{b\left(e^{b}-e^{-\lambda}\right)}\right) .
\end{aligned}
$$

После замены $e^{-\lambda}=q e^{1-q}\left(=p e^{1-p}\right), b=(p-q)^{2} / p$ константа $c_{-}^{*}$ принимает окончательный вид

$$
c_{-}^{*}(p, q)=\frac{(1-q) q e^{1-q}}{\sqrt{2 \pi}\left(1-q e^{1-q}\right)}\left(\frac{1}{1-q e^{1-q}}-\frac{p\left(\exp \left\{(p-q)^{2} / p\right\}-1\right)}{(p-q)^{2}\left(\exp \left\{(p-q)^{2} / p\right\}-q e^{1-q}\right)}\right) .
$$

Замечание 1. Нетрудно убедиться в том, что $c_{-}^{*}>0$. Действительно, для выражения из последней скобки в (22) справедлива оценка снизу

$$
\begin{aligned}
& \frac{1}{1-e^{-\lambda}}-\frac{e^{b}-1}{b\left(e^{b}-e^{-\lambda}\right)} \\
& \quad=\frac{1}{1-e^{-\lambda}}-\frac{e^{b}-1}{b e^{b}\left(1-e^{-\lambda-b}\right)}>\frac{1}{1-e^{-\lambda}}-\frac{e^{b}-1}{b e^{b}\left(1-e^{-\lambda}\right)} \\
& \quad=\frac{1}{1-e^{-\lambda}}\left(1-\frac{1-e^{-b}}{b}\right)>0
\end{aligned}
$$

так как $e^{-b}>1-b$.

Определим при $z \geq 0$ следующие функции $\left(0^{0}=1 !\right)$ :

$$
\begin{gathered}
\varphi(z)=\sum_{k=0}^{\infty} \frac{\left(q e^{-q}\right)^{k}(z+k)^{k}}{k !}, \\
J_{k}(z)=\frac{\left(p e^{-p}\right)^{k}(z+k)^{k}}{k !} e^{-p z}, \quad k=0,1, \ldots, \\
J(z)=\sum_{k=0}^{\infty} J_{k}(z)=e^{-p z} \sum_{k=0}^{\infty} \frac{\left(p e^{-p}\right)^{k}(z+k)^{k}}{k !},
\end{gathered}
$$

где, напомним, $p$ и $q$ удовлетворяют соотношениям (8)-(9); следовательно,

$$
J(z)=e^{-p z} \varphi(z) .
$$


Лемма 4. Справедлива формула

$$
\varphi(z)=\sum_{k=0}^{\infty} \frac{\left(q e^{-q}\right)^{k} \varphi(k)}{k !} z^{k} .
$$

Доказательство. Применяя в (23) формулу бинома Ньютона, находим

$$
\begin{aligned}
\varphi(z) & =\sum_{k=0}^{\infty} \frac{\left(q e^{-q}\right)^{k}}{k !} \sum_{m=0}^{k} C_{k}^{m} z^{m} k^{k-m} \\
& =\sum_{m=0}^{\infty} \frac{\left(q e^{-q}\right)^{m}}{m !} z^{m} \sum_{k=m}^{\infty} \frac{\left(q e^{-q}\right)^{k-m} k^{k-m}}{(k-m) !} \\
& =\sum_{m=0}^{\infty} \frac{\left(q e^{-q}\right)^{m}}{m !} z^{m} \sum_{k=0}^{\infty} \frac{\left(q e^{-q}\right)^{k}(m+k)^{k}}{k !} .
\end{aligned}
$$

Последний ряд, очевидно, равен $\varphi(m)$.

Следующее утверждение представляет и самостоятельный интерес.

Лемма 5. Если константы р и q связаны соотношениями (8), то для функций $\varphi(z)$ и $J(z)$ из (23) и (24) верны представления

$$
\begin{aligned}
& \varphi(z)=e^{q z} /(1-q), \\
& J(z)=e^{-(p-q) z} /(1-q) .
\end{aligned}
$$

Доказательство. Формальное дифференцирование ряда $\varphi(z)$ из (23)

$$
\varphi^{\prime}(z)=\sum_{k=1}^{\infty} \frac{\left(q e^{-q}\right)^{k}(z+k)^{k-1}}{(k-1) !}=q e^{-q} \sum_{k=0}^{\infty} \frac{\left(q e^{-q}\right)^{k}(z+1+k)^{k}}{k !}
$$

приводит к формуле

$$
\varphi^{\prime}(z)=q e^{-q} \varphi(z+1),
$$

справедливость которой вытекает из следующей оценки ряда в (27):

$$
\begin{aligned}
\sum_{k=0}^{\infty} \frac{\left(q e^{-q}\right)^{k}(z+1+k)^{k}}{k !} & =\sum_{k=0}^{\infty} \frac{\left(q e^{-q}\right)^{k} k^{k}(1+(z+1) / k)^{k}}{k !} \\
& <\sum_{k=0}^{\infty} \frac{\left(q e^{-q}\right)^{k} k^{k} e^{z+1}}{\sqrt{2 \pi} k^{k+1 / 2} e^{-k}}=\frac{e^{z+1}}{\sqrt{2 \pi}} \sum_{k=0}^{\infty} \frac{e^{-\lambda k}}{\sqrt{k}}
\end{aligned}
$$

где, напомним, $\lambda=q-1-\ln q($ см. (4), (11)).

Докажем сначала методом математической индукции первое равенство из (26) для целых значений аргумента:

$$
\varphi(n)=e^{q n} /(1-q), \quad n=0,1, \ldots
$$


Начало индукции базируется на известном из [12, с. 707 , формула (4)] ряде

$$
\sum_{k=1}^{\infty} \frac{\left(q e^{-q}\right)^{k} k^{k}}{k !}=\frac{q}{1-q}, \quad 0<q<1 .
$$

Отсюда и из (23) находим

$$
\varphi(0)=1+\sum_{k=1}^{\infty} \frac{\left(q e^{-q}\right)^{k} k^{k}}{k !}=1+\frac{q}{1-q}=\frac{1}{1-q},
$$

что соответствует формуле $(29)$ при $n=0$. Предполагая теперь, что равенство (29) верно для некоторого натурального числа $n$, из (28) получаем правильный индуктивный вывод:

$$
\varphi(n+1)=\frac{\varphi^{\prime}(n)}{q e^{-q}}=\frac{q e^{q n}}{(1-q) q e^{-q}}=\frac{e^{q(n+1)}}{(1-q)} .
$$

Для завершения доказательства первого равенства из (26) подставим выражение $(29)$ в представление для функции $\varphi(z)$ из леммы 4 :

$$
\varphi(z)=\sum_{k=0}^{\infty} \frac{\left(q e^{-q}\right)^{k} e^{q k} /(1-q)}{k !} z^{k}=\frac{1}{1-q} \sum_{k=0}^{\infty} \frac{(q z)^{k}}{k !}=\frac{e^{q z}}{1-q} .
$$

Отсюда и из (25) вытекает второе равенство из (26).

Лемма 6. Для последовательности функций $\left\{J_{k}(z)\right\}$ из (24) верны равенства

$$
z \sum_{k=0}^{\infty} \frac{J_{k}(z)}{z+k}=(1-q) J(z)=e^{-(p-q) z}, \quad z \geq 0 .
$$

Доказательство. Проверим сначала справедливость равенства

$$
z \sum_{k=0}^{\infty} \frac{J_{k}(z)}{z+k}=J(z)-p J(z+1) .
$$

Действительно,

$$
\begin{aligned}
\sum_{k=0}^{\infty} \frac{z}{z+k} J_{k}(z) & =\sum_{k=0}^{\infty} J_{k}(z)-\sum_{k=0}^{\infty} \frac{k}{z+k} J_{k}(z) \\
& =J(z)-\sum_{k=1}^{\infty} \frac{\left(p e^{-p}\right)^{k}(z+k)^{k-1}}{(k-1) !} e^{-p z} \\
& =J(z)-p \sum_{k=0}^{\infty} \frac{\left(p e^{-p}\right)^{k}(z+1+k)^{k}}{k !} e^{-p(z+1)} \\
& =J(z)-p J(z+1) .
\end{aligned}
$$


Воспользовавшись вторым равенством из (26) и соотношением (9), находим

$$
p J(z+1)=p e^{-(p-q)} J(z)=p e^{-p} e^{q} J(z)=q J(z) .
$$

Отсюда, а также из (30) и (26) получаем утверждение леммы.

Следующие результаты имеют отношение к интегралу из (13).

Лемма 7. Для любого натурального числа $n$ верно представление

$$
\begin{array}{r}
1-G^{*}(n)=(p-q)\left(\int_{0}^{\infty} \sum_{k=1}^{n} \frac{z+k}{z+n} \psi(z+k) J_{n-k}(z+k) d z\right. \\
\left.+\int_{0}^{\infty} z \psi(z) \sum_{k=n}^{\infty} \frac{J_{k}(z)}{z+k} d z\right) .
\end{array}
$$

Доказательство. Из (13) и леммы 1 следует, что

$$
1-G^{*}(n)=(p-q) \int_{n}^{\infty} e^{-p z} \sum_{k=0}^{[z]} \frac{p^{k} z^{k-1}}{k !}(z-k) \psi(z-k) d z=(p-q) I_{n}
$$

где через $I_{n}$ обозначен интеграл из (32). Тогда

$$
\begin{aligned}
I_{n} & =\sum_{m=n}^{\infty} \int_{m}^{m+1} e^{-p z} \sum_{k=0}^{m} \frac{p^{k} z^{k-1}}{k !}(z-k) \psi(z-k) d z \\
& =\sum_{m=n}^{\infty} \sum_{k=0}^{m} \int_{m}^{m+1}(z-k) \psi(z-k) \frac{p^{k} z^{k-1}}{k !} e^{-p z} d z .
\end{aligned}
$$

Преобразуем последнее выражение, пропуская в выкладках (ради простоты) подынтегральную функцию:

$$
\begin{aligned}
I_{n} & =\sum_{m=n}^{\infty} \sum_{k=0}^{m} \int_{m}^{m+1}=\left(\sum_{m=0}^{\infty}-\sum_{m=0}^{n-1}\right) \sum_{k=0}^{m} \int_{m}^{m+1} \\
& =\sum_{m=0}^{\infty} \sum_{k=0}^{m} \int_{m}^{m+1}-\sum_{m=0}^{n-1} \sum_{k=0}^{m} \int_{m}^{m+1} \\
& =\sum_{k=0}^{\infty} \sum_{m=k}^{\infty} \int_{m}^{m+1}-\sum_{k=0}^{n-1} \sum_{m=k}^{n-1} \int_{m}^{m+1} \\
& =\sum_{k=0}^{\infty} \int_{k}^{\infty}-\sum_{k=0}^{n-1} \int_{k}^{n}=\sum_{k=0}^{n-1} \int_{n}^{\infty}+\sum_{k=n}^{\infty} \int_{k}^{\infty}
\end{aligned}
$$


Таким образом,

$$
\begin{aligned}
I_{n}= & \sum_{k=0}^{n-1} \int_{n}^{\infty}(z-k) \psi(z-k) \frac{p^{k} z^{k-1}}{k !} e^{-p z} d z \\
& +\sum_{k=n}^{\infty} \int_{k}^{\infty}(z-k) \psi(z-k) \frac{p^{k} z^{k-1}}{k !} e^{-p z} d z .
\end{aligned}
$$

Первая сумма в (33) после замены $z:=z-n$ в интегралах преобразуется к виду

$$
\begin{gathered}
\sum_{k=0}^{n-1} \int_{0}^{\infty} \frac{z+n-k}{z+n} \psi(z+n-k) J_{k}(z+n-k) d z \\
=\int_{0}^{\infty} \sum_{k=1}^{n} \frac{z+k}{z+n} \psi(z+k) J_{n-k}(z+k) d z,
\end{gathered}
$$

а ряд из интегралов в (33) после замены $z:=z-k$ приводится к интегралу

$$
\sum_{k=n}^{\infty} \int_{0}^{\infty} z \psi(z) \frac{J_{k}(z)}{z+k} d z=\int_{0}^{\infty} z \psi(z) \sum_{k=n}^{\infty} \frac{J_{k}(z)}{z+k} d z .
$$

Отсюда, а также из (34), (33) и (32) получаем утверждение (31).

Замечание 2. Интеграл $I_{n}$ из (32) при $n=0$ представляется как

$$
I_{0}=\int_{0}^{\infty} e^{-p z} \sum_{k=0}^{[z]} \frac{p^{k} z^{k-1}}{k !}(z-k) \psi(z-k) d z=\frac{(1-q) p}{(p-q)^{2}}
$$

В самом деле, из представления (33) и леммы 6 находим

$$
\begin{aligned}
I_{0} & =\sum_{k=0}^{\infty} \int_{k}^{\infty}(z-k) \psi(z-k) \frac{p^{k} z^{k-1}}{k !} e^{-p z} d z \\
& =\sum_{k=0}^{\infty} \int_{0}^{\infty} z \psi(z) \frac{J_{k}(z)}{z+k} d z=\int_{0}^{\infty} z \psi(z) \sum_{k=0}^{\infty} \frac{J_{k}(z)}{z+k} d z \\
& =\int_{0}^{\infty} \psi(z) e^{-(p-q) z} d z=\frac{(1-q) p}{(p-q)^{2}},
\end{aligned}
$$

где последнее равенство вытекает из [10, лемма 4]:

$$
\int_{0}^{\infty} \psi(z) e^{-\beta z} d z=\frac{(1-q) p}{(p-q) \beta}
$$


если учесть, что $\beta=p-q$ согласно лемме 1 (см. также лемму 9 ниже). В силу (35) и (13) приходим к ожидаемому результату:

$$
G^{*}(+\infty)=\frac{(p-1) q}{p-q}+(p-q) \frac{(1-q) p}{(p-q)^{2}}=1 .
$$

Следующие две леммы определяют точную асимптотику интегралов из (31) при $n \rightarrow \infty$.

Лемма 8. При $n \rightarrow \infty$ имеем

$$
\int_{0}^{\infty} \sum_{k=1}^{n} \frac{z+k}{z+n} \psi(z+k) J_{n-k}(z+k) d z \sim \frac{c_{+}^{(1)}}{n^{3 / 2}} e^{-\lambda n}
$$

где

$$
c_{+}^{(1)}=\frac{1}{\sqrt{2 \pi}} \int_{0}^{\infty} \sum_{k=1}^{\infty} \frac{(z+k) \psi(z+k)}{p^{k}} e^{-(p-1) z} d z .
$$

Для константы $c_{+}^{(1)}$ справедлива оценка сверху

$$
c_{+}^{(1)}<\frac{p+1}{\sqrt{2 \pi}(p-1)^{3}} .
$$

Доказательство. Оценка (38) вытекает из неравенства $0<\psi(\cdot)<1$ для функции распределения $\psi(\cdot)$ из $(16)$ :

$$
\begin{aligned}
c_{+}^{(1)} & <\frac{1}{\sqrt{2 \pi}} \int_{0}^{\infty} \sum_{k=1}^{\infty} \frac{z+k}{p^{k}} e^{-(p-1) z} d z \\
& =\frac{1}{\sqrt{2 \pi}} \sum_{k=1}^{\infty} p^{-k} \int_{0}^{\infty} z e^{-(p-1) z} d z+\frac{1}{\sqrt{2 \pi}} \sum_{k=1}^{\infty} k p^{-k} \int_{0}^{\infty} e^{-(p-1) z} d z \\
& =\frac{1}{\sqrt{2 \pi}(p-1)^{2}} \sum_{k=1}^{\infty} p^{-k}+\frac{1}{\sqrt{2 \pi}(p-1)} \sum_{k=1}^{\infty} k p^{-k} \\
& =\frac{p+1}{\sqrt{2 \pi}(p-1)^{3}},
\end{aligned}
$$

так как

$$
\sum_{k=1}^{\infty} p^{-k}=1 /(p-1), \quad \sum_{k=1}^{\infty} k p^{-k}=p /(p-1)^{2} .
$$

Обозначим через $f_{n}(z)$ подынтегральную функцию из (36) и преобразуем ее, воспользовавшись определением функций $J_{k}(z)$ из $(24)$ : 
Обозначим

$$
\begin{aligned}
f_{n}(z) & =\sum_{k=1}^{n} \frac{z+k}{z+n} \psi(z+k) J_{n-k}(z+k) \\
& =\sum_{k=1}^{\infty} \frac{(z+k) \psi(z+k)\left(p e^{-p}\right)^{n-k}(z+n)^{n-k-1}}{(n-k) !} e^{-p(z+k)} \\
& =\frac{\left(p e^{-p}\right)^{n} n^{n-1}}{n !} e^{-p z}\left(1+\frac{z}{n}\right)^{n-1} \sum_{k=1}^{n} \frac{(z+k) \psi(z+k) n !}{p^{k}(n-k) !(z+n)^{k}}
\end{aligned}
$$

$$
\begin{aligned}
a_{n} & =\frac{\left(p e^{-p}\right)^{n} n^{n-1}}{n !}, \\
B_{n}^{k}(z) & = \begin{cases}\frac{n !}{(n-k) !(z+n)^{k}}, & k \leq n, \\
0, & k>n,\end{cases}
\end{aligned}
$$

где $k, n=1,2, \ldots, z \geq 0$. Тогда из (40) и (41) получаем представление

$$
\frac{f_{n}(z)}{a_{n}}=e^{-p z}\left(1+\frac{z}{n}\right)^{n-1} \sum_{k=1}^{\infty} \frac{(z+k) \psi(z+k)}{p^{k}} B_{n}^{k}(z) .
$$

Асимптотика выражений из (41) при $n \rightarrow \infty$ имеет вид

$$
\begin{gathered}
a_{n} \sim \frac{\left(p e^{-p}\right)^{n} n^{n-1}}{\sqrt{2 \pi} n^{n+1 / 2} e^{-n}}=\frac{e^{-\lambda n}}{\sqrt{2 \pi} n^{3 / 2}}, \\
\lim _{n \rightarrow \infty} B_{n}^{k}(z)=\lim _{n \rightarrow \infty} \frac{(1-1 / n) \cdots(1-(k-1) / n)}{(1+z / n)^{k}}=1,
\end{gathered}
$$

причем $B_{n}^{k}(z) \leq 1$ при всех значениях $k, n=1,2, \ldots$ и $z \geq 0$. Ввиду (43) и (39) заключаем, что у последовательности функций $f_{n}(z) / a_{n}$ из существуют не зависящая от $n$ интегрируемая мажоранта и предел:

$$
\begin{aligned}
\frac{f_{n}(z)}{a_{n}} \leq & e^{-(p-1) z} \sum_{k=1}^{\infty} \frac{(z+k)}{p^{k}}=e^{-(p-1) z}\left(\frac{z}{p-1}+\frac{p}{(p-1)^{2}}\right), \\
& \lim _{n \rightarrow \infty} \frac{f_{n}(z)}{a_{n}}=e^{-(p-1) z} \sum_{k=1}^{\infty} \frac{(z+k) \psi(z+k)}{p^{k}}
\end{aligned}
$$

Тогда из (44) следует, что

$$
\begin{aligned}
\lim _{n \rightarrow \infty} \frac{\int_{0}^{\infty} f_{n}(z) d z}{a_{n}} & =\int_{0}^{\infty} \lim _{n \rightarrow \infty}\left(\frac{f_{n}(z)}{a_{n}}\right) d z \\
& =\int_{0}^{\infty} e^{-(p-1) z} \sum_{k=1}^{\infty} \frac{(z+k) \psi(z+k)}{p^{k}} d z
\end{aligned}
$$

Отсюда и из первого соотношения в (43) получаем (36)-(37). 
Асимптотика второго интеграла из (31) представлена в следующей леммe.

Лемма 9. При $n \rightarrow \infty$ имеем

$$
\int_{0}^{\infty} z \psi(z) \sum_{k=n}^{\infty} \frac{J_{k}(z)}{z+k} d z \sim \frac{c_{+}^{(2)}}{n^{3 / 2}} e^{-\lambda n},
$$

где

$$
c_{+}^{(2)}=\frac{1}{\sqrt{2 \pi}\left(1-p e^{-p}\right)} \int_{0}^{\infty} z \psi(z) e^{-(p-1) z} d z .
$$

Для константы $c_{+}^{(2)}$ справедлива оценка сверху

$$
c_{+}^{(2)}<\frac{1}{\sqrt{2 \pi}\left(1-p e^{1-p}\right)(p-1)^{2}} .
$$

Доказательство. Обозначим

$$
g_{n}(z)=\sum_{k=n}^{\infty} \frac{J_{k}(z)}{z+k}, \quad n=1,2, \ldots .
$$

Преобразуем последовательность функций из (47), используя определение (24):

$$
\begin{aligned}
g_{n}(z) & =\sum_{k=n}^{\infty} \frac{\left(p e^{-p}\right)^{k}(z+k)^{k-1}}{k !} e^{-p z} \\
& =\sum_{k=0}^{\infty} \frac{\left(p e^{-p}\right)^{k+n}(z+k+n)^{k+n-1}}{(k+n) !} e^{-p z} \\
& =\frac{\left(p e^{-p}\right)^{n} e^{n}}{n^{3 / 2}} \sum_{k=0}^{\infty} \frac{\left(p e^{-p}\right)^{k} n^{3 / 2}(k+n)^{k+n-1}(1+z /(k+n))^{k+n-1}}{(k+n) ! e^{n}} e^{-p z}
\end{aligned}
$$

Отсюда получаем представление

$$
g_{n}(z)=\frac{e^{-\lambda n}}{n^{3 / 2}} \sum_{k=0}^{\infty} C_{n}^{k}(z),
$$

где

$$
\begin{gathered}
C_{n}^{k}(z)=\frac{\left(p e^{-p}\right)^{k} n^{3 / 2}(k+n)^{k+n-1}(1+z /(k+n))^{k+n-1}}{(k+n) ! e^{n}} e^{-p z}, \\
k=0,1, \ldots, \quad n=1,2, \ldots
\end{gathered}
$$


Тогда в силу (47) и (48) интеграл из (45) принимает вид

$$
\int_{0}^{\infty} z \psi(z) \sum_{k=n}^{\infty} \frac{J_{k}(z)}{z+k} d z=\frac{e^{-\lambda n}}{n^{3 / 2}} \int_{0}^{\infty} z \psi(z) \sum_{k=0}^{\infty} C_{n}^{k}(z) d z .
$$

Заметим, что

$$
\begin{aligned}
C_{n}^{k}(z) & \leq \frac{\left(p e^{-p}\right)^{k} n^{3 / 2}(k+n)^{k+n-1} e^{z}}{\sqrt{2 \pi}(k+n)^{k+n+1 / 2} e^{-k-n} e^{n}} e^{-p z} \\
& =\frac{e^{-\lambda k}}{\sqrt{2 \pi}}\left(\frac{n}{k+n}\right)^{3 / 2} e^{-(p-1) z} \leq \frac{e^{-\lambda k}}{\sqrt{2 \pi}} e^{-(p-1) z}, \quad k=0,1, \ldots,
\end{aligned}
$$

и при этом

$$
\lim _{n \rightarrow \infty} C_{n}^{k}(z)=\frac{e^{-\lambda k}}{\sqrt{2 \pi}} e^{-(p-1) z}, \quad k=0,1, \ldots
$$

Последние обстоятельства являются основанием для предельного перехода:

$$
\begin{aligned}
\lim _{n \rightarrow \infty} & \int_{0}^{\infty} z \psi(z) \sum_{k=0}^{\infty} C_{n}^{k}(z) d z \\
& =\int_{0}^{\infty} z \psi(z) \sum_{k=0}^{\infty} \lim _{n \rightarrow \infty} C_{n}^{k}(z) d z \\
& =\frac{1}{\sqrt{2 \pi}} \sum_{k=0}^{\infty} e^{-\lambda k} \int_{0}^{\infty} z \psi(z) e^{-(p-1) z} d z \\
& =\frac{1}{\sqrt{2 \pi}\left(1-e^{-\lambda}\right)} \int_{0}^{\infty} z \psi(z) e^{-(p-1) z} d z=c_{+}^{(2)},
\end{aligned}
$$

где последнее равенство является следствием определений (4) и (11):

$$
e^{-\lambda}=p e^{1-p}\left(=q e^{1-q}\right) .
$$

Отсюда и из (49) получаем утверждение (45).

Оценка сверху для константы $c_{+}^{(2)}$ вытекает из (46) и неравенства

$$
c_{+}^{(2)}<\frac{1}{\sqrt{2 \pi}\left(1-p e^{-p}\right)} \int_{0}^{\infty} z e^{-(p-1) z} d z=\frac{1}{\sqrt{2 \pi}\left(1-p e^{1-p}\right)(p-1)^{2}} .
$$

Лемма 10. Для любого $s>0$ имеем

$$
\int_{0}^{\infty} \psi(z) e^{-s z} d z=\frac{1-q}{s-q+q e^{-s}}
$$




$$
\int_{0}^{\infty} z \psi(z) e^{-s z} d z=\frac{(1-q)\left(1-q e^{-s}\right)}{\left(s-q+q e^{-s}\right)^{2}}
$$

где функция $\psi(z)$ определена в (16).

Доказательство. Воспользуемся свойствами функции $\psi(z)$ (см. [10, лемма 2]):

$$
\begin{aligned}
\psi^{\prime}(z) & =q(\psi(z)-\psi(z-1)), & & z \geq 1, \\
\psi(0) & =1-q, \quad \psi(z)=0, & & z<0,
\end{aligned}
$$

при интегрировании по частям:

$$
\begin{aligned}
I(s) & =\int_{0}^{\infty} \psi(z) e^{-s z} d z=-\frac{1}{s} \int_{0}^{\infty} \psi(z) d e^{-s z} \\
& =\frac{1-q}{s}+\frac{q}{s} \int_{0}^{\infty}(\psi(z)-\psi(z-1)) e^{-s z} d z \\
& =\frac{1-q}{s}+\frac{q}{s} I(s)-\frac{q}{s} \int_{1}^{\infty} \psi(z-1) e^{-s z} d z \\
& =\frac{1-q}{s}+\frac{q}{s} I(s)-\frac{q e^{-s}}{s} I(s) .
\end{aligned}
$$

Разрешая уравнение относительно $I(s)$, приходим к (50). Аналогично доказывается и равенство (51), в доказательстве которого используются то же самое обозначение $I(s)$, но уже для интеграла из (51):

$$
\begin{aligned}
I(s) & =\int_{0}^{\infty} z \psi(z) e^{-s z} d z=-\frac{1}{s} \int_{0}^{\infty} z \psi(z) d e^{-s z} \\
& =\frac{1}{s} \int_{0}^{\infty} e^{-s z} d z \psi(z) \\
& =\frac{1}{s} \int_{0}^{\infty} \psi(z) e^{-s z} d z+\frac{q}{s} \int_{0}^{\infty} z(\psi(z)-\psi(z-1)) e^{-s z} d z \\
& =\frac{1}{s} \int_{0}^{\infty} \psi(z) e^{-s z} d z+\frac{q}{s} I(s)-\frac{q}{s} \int_{1}^{\infty} z \psi(z-1) e^{-s z} d z .
\end{aligned}
$$

Последний интеграл здесь равен

$$
\int_{0}^{\infty}(z+1) \psi(z) e^{-s(z+1)} d z=e^{-s} I(s)+e^{-s} \int_{0}^{\infty} \psi(z) e^{-s z} d z
$$

что вместе с предыдущей цепочкой равенств приводит к уравнению

$$
I(s)=\frac{1}{s} \int_{0}^{\infty} \psi(z) e^{-s z} d z+\frac{q}{s} I(s)-\frac{q e^{-s}}{s} I(s)-\frac{q e^{-s}}{s} \int_{0}^{\infty} \psi(z) e^{-s z} d z .
$$


Отсюда выражаем

$$
I(s)=\frac{1-q e^{-s}}{s-q+q e^{-s}} \int_{0}^{\infty} \psi(z) e^{-s z} d z
$$

через интеграл из (50).

Из (51) и (46) вытекает

Лемма 11. Константа $c_{+}^{(2)}$ из (45)-(46) имеет вид

$$
c_{+}^{(2)}=\frac{(1-q)\left(1-q e^{1-p}\right)}{\sqrt{2 \pi}\left(1-p e^{1-p}\right)\left(p-q-1+q e^{1-p}\right)^{2}} .
$$

Следующие леммы потребуются для нахождения константы $c_{+}^{(1)}$ из (37).

Лемма 12. Функции

$$
\begin{aligned}
& Q(z)=\sum_{k=1}^{\infty} \frac{\psi(z+k)}{p^{k}}, \quad Q(+\infty)=\frac{1}{p-1}, \\
& H(z)=\sum_{k=1}^{\infty} \frac{(z+k) \psi(z+k)}{p^{k}}, \quad z \geq 0,
\end{aligned}
$$

удовлетворяют следующим дифференциальным уравнениям:

$$
\begin{gathered}
Q^{\prime}(z)=\frac{q(p-1)}{p} Q(z)-\frac{q}{p} \psi(z), \\
H^{\prime}(z)=\frac{q(p-1)}{p} H(z)+\frac{p-q}{p} Q(z)-\frac{q}{p} z \psi(z)-\frac{q}{p} \psi(z),
\end{gathered}
$$

и при этом

$$
\begin{aligned}
& Q(0)=\sum_{k=1}^{\infty} \frac{\psi(k)}{p^{k}}=\frac{1-q}{p \exp \{q(1-p) / p\}-1} \\
& H(0)=\sum_{k=1}^{\infty} \frac{k \psi(k)}{p^{k}}=\frac{(1-q)(p-q) \exp \{q(1-p) / p\}}{(p \exp \{q(1-p) / p\}-1)^{2}}
\end{aligned}
$$

Доказательство. Ввиду того, что функция распределения $\psi(\cdot)$ ограничена вместе со своей производной (см. (52)), ряды (53)-(54) можно дифференцировать почленно. Применяя равенство (52) при дифференцировании ряда (53), приходим к линейному дифференциальному уравнению (55):

$$
Q^{\prime}(z)=q \sum_{k=1}^{\infty} \frac{\psi(z+k)}{p^{k}}-q \sum_{k=1}^{\infty} \frac{\psi(z+k-1)}{p^{k}}
$$




$$
\begin{aligned}
& =q Q(z)-\frac{q}{p} \sum_{k=0}^{\infty} \frac{\psi(z+k)}{p^{k}}=q Q(z)-\frac{q}{p} Q(z)-\frac{q}{p} \psi(z) \\
& =\frac{q(p-1)}{p} Q(z)-\frac{q}{p} \psi(z) .
\end{aligned}
$$

Подставляя функцию

$$
Q(z)=\frac{q}{p} \int_{0}^{\infty} \psi(z+t) \exp \left\{-\frac{q(p-1)}{p} t\right\} d t
$$

в равенство (55), убеждаемся, что эта функция является решением линейного дифференциального уравнения (55):

$$
\begin{aligned}
Q^{\prime}(z)= & \frac{q}{p} \int_{0}^{\infty} \exp \left\{-\frac{q(p-1)}{p} t\right\} d \psi(z+t) \\
= & \left.\frac{q}{p} \psi(z+t) \exp \left\{-\frac{q(p-1)}{p} t\right\}\right|_{0} ^{\infty} \\
& \quad+\frac{q(p-1)}{p} \cdot \frac{q}{p} \int_{0}^{\infty} \psi(z+t) \exp \left\{-\frac{q(p-1)}{p} t\right\} d t \\
= & -\frac{q}{p} \psi(0)+\frac{q(p-1)}{p} Q(z)
\end{aligned}
$$

удовлетворяющим граничному условию из (53):

$$
Q(+\infty)=\frac{q}{p} \int_{0}^{\infty} \exp \left\{-\frac{q(p-1)}{p} t\right\} d t=\frac{1}{p-1}
$$

Следовательно, из формул (59) и (50) выводим формулу (57):

$$
Q(0)=\frac{q}{p} \int_{0}^{\infty} \psi(t) \exp \left\{-\frac{q(p-1)}{p} t\right\} d t=\frac{1-q}{p \exp \{q(1-p) / p\}-1},
$$

а дифференцирование ряда (57) по параметру $p$ приводит к формуле (58):

$$
\begin{aligned}
H(0) & =\sum_{k=1}^{\infty} \frac{k \psi(k)}{p^{k}}=-p\left(\sum_{k=1}^{\infty} \frac{\psi(k)}{p^{k}}\right)_{p}^{\prime} \\
& =-p\left(\frac{1-q}{p \exp \{q(1-p) / p\}-1}\right)_{p}^{\prime} \\
& =\frac{(1-q)(p-q) \exp \{q(1-p) / p\}}{(p \exp \{q(1-p) / p\}-1)^{2}} .
\end{aligned}
$$


Осталось проверить равенство (56). Из (54) и (52) находим

$$
\begin{aligned}
H^{\prime}(z) & =\sum_{k=1}^{\infty} \frac{\psi(z+k)}{p^{k}}+q \sum_{k=1}^{\infty} \frac{(z+k)(\psi(z+k)-\psi(z+k-1))}{p^{k}} \\
& =Q(z)+q H(z)-\frac{q}{p} \sum_{k=1}^{\infty} \frac{(z+k) \psi(z+k-1)}{p^{k-1}} \\
& =Q(z)+q H(z)-\frac{q}{p} \sum_{k=0}^{\infty} \frac{(z+k+1) \psi(z+k)}{p^{k}} \\
& =Q(z)+q H(z)-\frac{q}{p} \sum_{k=1}^{\infty} \frac{(z+k+1) \psi(z+k)}{p^{k}}-\frac{q}{p}(z+1) \psi(z) \\
& =Q(z)+q H(z)-\frac{q}{p} H(z)-\frac{q}{p} Q(z)-\frac{q}{p} z \psi(z)-\frac{q}{p} \psi(z) \\
& =\frac{q(p-1)}{p} H(z)+\frac{p-q}{p} Q(z)-\frac{q}{p} z \psi(z)-\frac{q}{p} \psi(z) .
\end{aligned}
$$

Лемма 13. Для функции $Q(z)$ из (53) верна формула

$$
\begin{aligned}
A & :=\int_{0}^{\infty} Q(z) e^{-(p-1) z} d z \\
& =\frac{1-q}{(p-1)(p-q)}\left(\frac{p}{p \exp \{q(1-p) / p\}-1}-\frac{q}{p-q-1+q e^{1-p}}\right) .
\end{aligned}
$$

Доказательство. Воспользуемся формулой (55) при интегрировании по частям:

$$
\begin{aligned}
A & =-\frac{1}{p-1} \int_{0}^{\infty} Q(z) d e^{-(p-1) z}=\frac{Q(0)}{p-1}+\frac{1}{p-1} \int_{0}^{\infty} Q^{\prime}(z) e^{-(p-1) z} d z \\
& =\frac{Q(0)}{p-1}+\frac{q}{p} A-\frac{q}{p(p-1)} \int_{0}^{\infty} \psi(z) e^{-(p-1) z} d z,
\end{aligned}
$$

а значит,

$$
A=\frac{p Q(0)}{(p-1)(p-q)}-\frac{q}{(p-1)(p-q)} \int_{0}^{\infty} \psi(z) e^{-(p-1) z} d z .
$$

Осталось воспользоваться формулами (50) и (57).

Лемма 14. Константа $c_{+}^{(1)}$ из (36)-(37) представляется как

$$
\begin{gathered}
c_{+}^{(1)}=\frac{1-q}{\sqrt{2 \pi}(p-1)^{2}(p-q)}\left(\frac{p\left(\left(p^{2}-p q+q\right) \exp \{q(1-p) / p\}-1\right)}{(p \exp \{q(1-p) / p\}-1)^{2}}\right. \\
\left.-\frac{q\left(p^{2}-p q-1+q e^{1-p}\right)}{\left(p-q-1+q e^{1-p}\right)^{2}}\right) .
\end{gathered}
$$


Доказательство. Из формул (37) и (54) получаем

$$
c_{+}^{(1)}=\frac{1}{\sqrt{2 \pi}} \int_{0}^{\infty} H(z) e^{-(p-1) z} d z .
$$

Интеграл из (62) находим, используя равенство (56):

$$
\begin{aligned}
B:= & \int_{0}^{\infty} H(z) e^{-(p-1) z} d z \\
= & -\frac{1}{p-1} \int_{0}^{\infty} H(z) d e^{-(p-1) z} \\
= & \frac{H(0)}{p-1}+\frac{1}{p-1} \int_{0}^{\infty} H^{\prime}(z) e^{-(p-1) z} d z \\
= & \frac{H(0)}{p-1}+\frac{q}{p} B+\frac{p-q}{p(p-1)} \int_{0}^{\infty} Q(z) e^{-(p-1) z} d z \\
& \quad-\frac{q}{p(p-1)} \int_{0}^{\infty} z \psi(z) e^{-(p-1) z} d z-\frac{q}{p(p-1)} \int_{0}^{\infty} \psi(z) e^{-(p-1) z} d z .
\end{aligned}
$$

Отсюда следует, что

$$
\begin{aligned}
B= & \int_{0}^{\infty} H(z) e^{-(p-1) z} d z \\
= & \frac{p H(0)}{(p-1)(p-q)}+\frac{1}{p-1} \int_{0}^{\infty} Q(z) e^{-(p-1) z} d z \\
& \quad-\frac{q}{(p-1)(p-q)} \int_{0}^{\infty} z \psi(z) e^{-(p-1) z} d z-\frac{q}{(p-1)(p-q)} \int_{0}^{\infty} \psi(z) e^{-(p-1) z} d z .
\end{aligned}
$$

Подставляя сюда выражения для интегралов, найденные в леммах 10 и 13, а также выражение (58), находим

$$
\begin{aligned}
\int_{0}^{\infty} H(z) e^{-(p-1) z} d z= & \frac{p(1-q) \exp \{q(1-p) / p\}}{(p-1)(p \exp \{q(1-p) / p\}-1)^{2}} \\
& +\frac{p(1-q)}{(p-1)^{2}(p-q)(p \exp \{q(1-p) / p\}-1)} \\
& -\frac{q(1-q)}{(p-1)^{2}(p-q)\left(p-q-1+q e^{1-p}\right)} \\
& -\frac{q(1-q)\left(1-q e^{1-p}\right)}{(p-1)(p-q)\left(p-q-1+q e^{1-p}\right)^{2}} \\
& -\frac{q(1-q)}{(p-1)(p-q)\left(p-q-1+q e^{1-p}\right)} .
\end{aligned}
$$


После приведения к общему знаменателю положительных, а затем и отрицательных дробей, приходим к результату:

$$
\begin{gathered}
\int_{0}^{\infty} H(z) e^{-(p-1) z} d z=\frac{p(1-q)\left(\left(p^{2}-p q+q\right) \exp \{q(1-p) / p\}-1\right)}{(p-1)^{2}(p-q)(p \exp \{q(1-p) / p\}-1)^{2}} \\
-\frac{q(1-q)\left(p^{2}-p q-1+q e^{1-p}\right)}{(p-1)^{2}(p-q)\left(p-q-1+q e^{1-p}\right)^{2}} .
\end{gathered}
$$

Отсюда и из (62) находим константу $c_{+}^{(1)}$.

Доказательство теоремы 2. Из представления (31) и асимптотических формул (36) и (45) получаем асимптотическое выражение

$$
1-G^{*}(n) \sim(p-q) \frac{c_{+}^{(1)}+c_{+}^{(2)}}{n^{3 / 2}} e^{-\lambda n}, \quad n \rightarrow \infty .
$$

Подставляя сюда найденные в леммах 11 и 14 константы $c_{+}^{(1)}$ и $c_{+}^{(2)}$, находим

$$
\begin{gathered}
c_{+}^{*}=\frac{(p-q)}{\sqrt{2 \pi}}\left(\frac{p(1-q)\left(\left(p^{2}-p q+q\right) \exp \{q(1-p) / p\}-1\right)}{(p-1)^{2}(p-q)(p \exp \{q(1-p) / p\}-1)^{2}}\right. \\
-\frac{q(1-q)\left(p^{2}-p q-1+q e^{1-p}\right)}{(p-1)^{2}(p-q)\left(p-q-1+q e^{1-p}\right)^{2}} \\
\left.+\frac{(1-q)\left(1-q e^{1-p}\right)}{\left(1-p e^{1-p}\right)\left(p-q-1+q e^{1-p}\right)^{2}}\right) .
\end{gathered}
$$

Сумма последних двух дробей приводится к виду

$$
\frac{p(1-q)\left(p-q-1+q e^{1-p}\right)^{2}}{(p-1)^{2}(p-q)\left(1-p e^{1-p}\right)\left(p-q-1+q e^{1-p}\right)^{2}}=\frac{p(1-q)}{(p-1)^{2}(p-q)\left(1-p e^{1-p}\right)} .
$$

Следовательно,

$$
c_{+}^{*}=\frac{p(1-q)}{\sqrt{2 \pi}(p-1)^{2}}\left(\frac{\left(p^{2}-p q+q\right) \exp \{q(1-p) / p\}-1}{(p \exp \{q(1-p) / p\}-1)^{2}}+\frac{1}{1-p e^{1-p}}\right) .
$$

Остается заметить, что выражение $p e^{1-p}$ в (63) можно заменить выражением $q e^{1-q}$.

В заключение выражаю глубокую благодарность И. С. Борисову за предложение заняться асимптотическим анализом функции распределения $G(x)$. Благодарю также рецензента статьи за полезные замечания. 


\section{Список литературы}

1. Борисов И. С., Миронов Д. В. Асимптотическое представление отношения правдоподобия для многомерных выборок с разрывными плотностями // ТВП. 2000. Т. 45, № 2. С. 345-356.

2. Борисов И. С., Миронов Д. В. Асимптотическое представление отношения правдоподобия для нерегулярных семейств распределений в многомерном случае // Сиб. матем. журн. 2001. Т. 42, № 2. С. 275-288.

3. Боровков А. А. Предельные теоремы о распределении максимума сумм ограниченных решетчатых случайных величин. I; II // ТВП. 1960. Т. 5, № 2. C. 137-171; T. 5, № 4. С. 377-392.

4. Боровков А. А. Новые предельные теоремы в граничных задачах для сумм независимых слагаемых // Сиб. матем. журн. 1962. Т. 3, № 5 . C. 645-694.

5. Боровков А. А. Об оценивании параметров в случае разрывных плотностей // ТВП. 2018. Т. 63, № 2. С. 211-239.

6. Ибрагимов И. А., Хасьминский Р. З. Асимптотическая теория оченивания. М.: Наука, 1979.

7. Мосягин В. Е. Оценка скорости сходимости распределения процесса максимального правдоподобия в нерегулярном случае // Cuб. матем. журн. 1991. Т. 32, № 4. С. 96-103.

8. Мосягин В. Е. Асимптотическое представление для процесса отношения правдоподобия в случае разрывной плотности // Сиб. матем. журн. 1994. Т. 35, № 2. С. 416-423.

9. Мосягин В. Е. Оценка скорости сходимости распределений нормированных оценок максимального правдоподобия в случае разрывной плотности // Сиб. матем. журн. 1996. Т. 37, №4. С. 895-903.

10. Мосягин В. Е., Швемлер Н. А. Распределение момента максимума разности двух пуассоновских процессов с отрицательным линейным сносом // Сиб. электрон. матем. изв. 2016. Т. 13. С. 1229-1248.

11. Мосягин В. Е., Швемлер Н. А. Локальные свойства предельного распределения статистической оценки точки разрыва плотности // Cuб. электрон. матем. изв. 2017. Т. 14. С. 1307-1316.

12. Прудников А. П., Брычков Ю. А., Маричев О. И. Интегралы и рядъ. Элементарные функиии. М.: Наука, 1981.

13. Ротарь В. И., Бенинг В. Е. Введение в математическую теорию страхования // Обозрение прикладной и промышленной математики. 1994. T. 1, № 5. C. 698-779. 
14. Скороход А. В. Случайные процессы с независимыми приращениями. М.: Наука, 1964.

Мосягин Вячеслав Евгеньевич

Тюменский гос. университет, ул. Володарского, 6,

Тюмень, 625003 РОССИЯ.

E-mail: vmosyagin@mail.ru
Поступила в редакцию 30 марта 2019 г.

Получена после доработки 21 апреля 2019 г.

Принята к публикации 10 июня 2019 г. 